\title{
Searches for New Particles/Phenomena at CDF
}

\author{
Hyunsoo Kim (for the CDF Collaboration) \\ University of Illinois at Urbana-Champaign, Urbana, IL 61801-3080, USA
}

Presented at the 14th Topical Conference on Hadron Collider Physics, HCP2002, Karlsruhe, Germany, 29 Sep.-4 Oct. 2002.

\section{Overview}

Recent results from the searches for new particles and phenomena using data collected by $\mathrm{CDF}$ are presented. Most results are from the data taken during 1994-95 period (Run I), but some preliminary results from the current data taking period (Run IIa) are presented as well.

\section{Searches for New Gauge Bosons}

A simple way to search for a $W^{\prime}$ boson is to look for $W^{\prime} \rightarrow e \nu$ and $\mu \nu$ decay channels, which would appear as an excess in the transverse mass distribution. These analyses required an electron (muon) with $E_{T}>25 \mathrm{GeV}$ $\left(p_{T}>20 \mathrm{GeV} / c\right)$ and $\not_{T}>25(20) \mathrm{GeV}[1,2]$. The transverse mass distributions were found to be consistent with the background prediction and, assuming the standard model strength couplings, the 95\% CL lower mass limits were set at $755 \mathrm{GeV} / c^{2}$ for the electron channel, $660 \mathrm{GeV} / c^{2}$ for the muon channel, and $786 \mathrm{GeV} / c^{2}$ for channels combined.

Also, the $W^{\prime}$ boson was searched for using the $W^{\prime} \rightarrow t \bar{b} \rightarrow b \bar{b} l \nu$ decay channel [3]. This channel, along with the dijet decay channel, provides the sensitivity to a case of very heavy right-handed neutrinos $\left(\nu_{R}\right), M_{W^{\prime}}<M_{\nu_{R}}$, where the leptonic decay channels of a $W^{\prime}$ boson are kinematically suppressed. This analysis required an electron (muon) with $E_{T}>20 \mathrm{GeV}$ $\left(p_{T}>20 \mathrm{GeV} / c\right), \mathbb{E}_{T}>20 \mathrm{GeV}$, and two or three jets with $E_{T}>15 \mathrm{GeV}$ with at least one of them to be identified as coming from a $b$-jet. The "l$\nu j j$ " invariant mass distribution was formed with a $l \nu$ invariant mass constrained to the $W$ boson mass. No excess over background expectation was observed in the distribution and the limits were set on the production cross section times branching fraction of $W^{\prime} \rightarrow t \bar{b}$ as a function of $M_{W^{\prime}}$ for the following two cases; $M_{W}^{\prime} \gg M_{\nu_{R}}$ and $M_{W}^{\prime}<M_{\nu_{R}}$. The $95 \%$ CL limits are shown in Fig. 1. For the standard model strength couplings, the mass region of $225<M_{W^{\prime}}<536(566) \mathrm{GeV} / c^{2}$ is excluded for $M_{W^{\prime}} \gg M_{\nu_{R}}\left(M_{W^{\prime}}<M_{\nu_{R}}\right)$ case. 


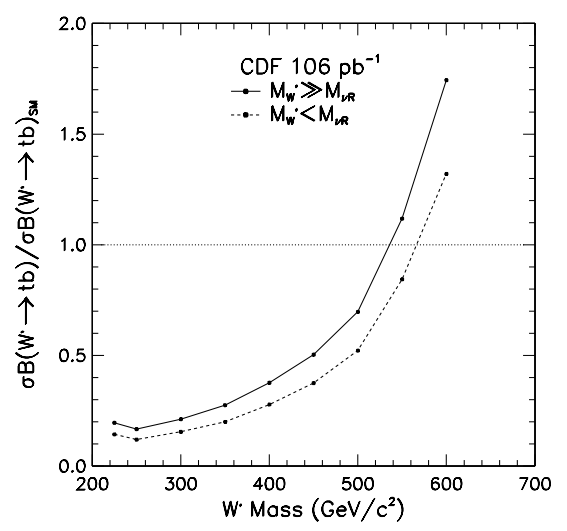

Fig. 1. The upper limits on the $W^{\prime}$ boson production cross section as a function of the $W^{\prime}$ boson mass. Limits are shown for the case $M_{W^{\prime}} \gg M_{\nu_{R}}$ (solid) and $M_{W^{\prime}}<M_{\nu_{R}}$ (dashed). The intercepts at $\frac{\sigma \mathcal{B}\left(W^{\prime} \rightarrow t b\right)}{\sigma \mathcal{B}\left(W^{\prime} \rightarrow t b\right)_{S M}}=1$ correspond to the $95 \%$ CL limits on the $W^{\prime}$ boson mass with Standard Model strength couplings

CDF searched for $Z^{\prime}$ boson production in the Drell-Yan channel in Run I [4]. This analysis required two isolated high $E_{T}\left(p_{T}\right)$, oppositely charged electrons (muons) and set a lower limit on the $Z^{\prime}$ mass at $690 \mathrm{GeV} / c^{2}$ at 95\% CL assuming the standard model strength couplings. In Run IIa, using $10 \mathrm{pb}^{-1}$ of electron data and $16 \mathrm{pb}^{-1}$ of muon data, CDF has set the limits at $450 \mathrm{GeV} / c^{2}$ and $270 \mathrm{GeV} / c^{2}$, respectively [5]. With the expected $2 \mathrm{fb}^{-1}$ of data accumulated by the end of Run IIa, $Z^{\prime}$ boson mass up to $1 \mathrm{TeV} / c^{2}$ can be excluded [6].

An analysis to search for the $Z^{\prime}$ boson from topcolor-assisted technicolor was performed by looking for resonance in the $t \bar{t}$ production [7]. This analysis looked for one $W$ boson from a top quark decaying leptonically and the other decaying hadronically. The analysis required a high $p_{T}$ electron or muon, a large $\not_{T}$, and four or more jets with at least one of them to be $b$-tagged. A kinematic fit was performed for wach event to determine the $t \bar{t}$ invariant mass value. The observed invariant mass distribution was found to be consistent with the standard model prediction and the 95\% CL lower limits on the leptophobic $Z^{\prime}$ boson mass was set at $780(480) \mathrm{GeV} / c^{2}$ for $\Gamma=0.04(0.012) M_{Z^{\prime}}$.

\section{$3 \quad$ Large Extra Dimensions}

The existence of the large extra dimension can manifest itself in $p \bar{p}$ collisions through exchanges of a Kaluza-Klein tower of gravitons, which subsequently produce a $l^{+} l^{-}$or $\gamma \gamma$ pair $[8,9]$. In Run I CDF searched for the graviton 
exchange processes in the dielectron and diphoton final states, looking for excess in the invariant mass distributions, and found no excess [10]. The $95 \%$ CL lower limits on the effective Planck scale in the extra dimensions, $M_{S}$, were set at $989(853) \mathrm{GeV}$ for $\Lambda_{\text {Hewett }}=-1(+1)[11]$.

The Randall-Sundrum graviton [12] was searched for using data collected in Run IIa, $10 \mathrm{pb}^{-1}$ for dielectron channel and $16 \mathrm{pb}^{-1}$ for dimuon channel. The $95 \%$ CL limits were set at $340 \mathrm{GeV} / c^{2}$ and $255 \mathrm{GeV} / c^{2}$ for dielectron and dimuon channels, respectively, for $k / M_{P l}=0.1$ [5].

Another way to search for the Large Extra Dimensions is to look for emission of a graviton and a photon or a jet recoiling against it, where the graviton does not interact with detector leaving a large $\not_{T}$ [9]. CDF has searched for photon-graviton final state using Run I data [13]. This analysis required a photon with $E_{T}>50 \mathrm{GeV}, E_{T}>40 \mathrm{GeV}$, and no jets and tracks with significant $E_{T}$ and $p_{T}$, respectively. The number of observed events was 11 and the number of expected background events was $11.0 \pm 2.2$. The main backgrounds are from cosmic rays and $Z_{\gamma} \rightarrow \nu \nu \gamma$. The $95 \%$ CL limits were set as $M_{S}>549 \mathrm{GeV}(n=4), 581 \mathrm{GeV}(n=6)$, and $602 \mathrm{GeV}(n=8)$, where $n$ is the number of extra dimensions.

\section{Search for Long-Lived Heavy Charged Particles}

A search for CHAMPs, heavy charged particles that are long-lived enough to leave the detector, was performed at CDF at Run I [14]. This analysis looked for high- $p_{T}$ track with a large ionisation loss in the detector. The observed number of events was consistent with the background prediction and a lower limit of 220 (190) $\mathrm{GeV} / c^{2}$ was set at 95\% CL for a long-lived up (down) type 4 th generation quark.

In Run II, CDF is developing an analysis employing the newly installed time-of-flight detector. This analysis searches a high- $p_{T}$ track with a large time-of-flight indicating a heavy slowly moving charged particle.

\section{Leptoquark Searches}

CDF searched for the direct production of three generations of leptoquarks using the data taken during Run I [15-17]. The 95\% CL lower mass limit of the first generation scalar leptoquark in the eejj decay channel with a branching ratio $\beta \equiv \mathcal{B}(L Q \rightarrow l q)=1$ was set at $213 \mathrm{GeV} / c^{2}$. The lower mass limits were set for the second generation scalar leptoquarks at $202 \mathrm{GeV} / c^{2}$, $164 \mathrm{GeV} / c^{2}$, and $122 \mathrm{GeV} / c^{2}$ for $\mu \mu j j(\beta=1), \mu \nu j j(\beta=0.5)$, and $\nu \nu c c$ $(\beta=0)$ decay channels, respectively. The limits on the second generation scalar leptoquarks were combined to give the lower mass limit of $183 \mathrm{GeV} / c^{2}$ at $\beta=0.5$. The lower mass limits on the third generation scalar leptoquarks are $99 \mathrm{GeV} / c^{2}$ and $149 \mathrm{GeV} / c^{2}$ for the $\tau \tau b b(\beta=1)$ and $\nu \nu b b(\beta=1)$ decay channels, respectively. 


\section{New Physics with Inclusive Lepton and Photon Final States}

Observation of a single ee $\gamma \gamma \mathbb{H}_{T}$ event at CDF in Run I data [18] prompted a search for anomalous production of events with a high $E_{T}$ photon and a lepton $(e$ or $\mu)$ in the final state [19]. Also, events with additional photons and leptons as well as large $\mathbb{H}_{T}$ were analysed. The search is based on $86 \mathrm{pb}^{-1}$ of data taken during Run I.

The results are shown in Table 1 . They were found to be consistent with the standard model expectations with a possible exception of $l \gamma \not_{T}$ where $7.6 \pm 0.7$ events were expected from the standard model and 16 events were observed.

Table 1. The results for the inclusive lepton and photon final state analysed. Shown here are the mean number of events predicted by the standard model, $\mu_{S M}$, the observed number of events, $n_{o}$, and the likelihood of the observation being consistent with $\mu_{S M}, P\left(n \geq n_{o} \mid \mu_{S M}\right)$

\begin{tabular}{lccc}
\hline Category & $\mu_{S M}$ & $n_{o} P\left(n \geq n_{o} \mid \mu_{S M}\right)$ \\
\hline Two-body $l \gamma X$ & $24.9 \pm 2.433$ & $9.3 \%$ \\
Multi-body $l \gamma X$ & $20.2 \pm 1.7$ & 27 & $10.0 \%$ \\
Multi-body $l \gamma X$ & $5.8 \pm 0.6$ & 33 & $68.0 \%$ \\
Multi-body $l \gamma \not_{T} X$ & $7.6 \pm 0.7$ & 16 & $0.7 \%$ \\
\hline
\end{tabular}

\section{Search for Gluinos and Scalar Quarks}

This analysis searched for the production and decay of gluinos and scalar quarks (squarks) in the $\geq 3$-jet events with large $\#_{T}$ at CDF [20]. The three or more jets are expected from the hadronic decays of the gluinos and/or squarks. The presence of large $\not_{T}$ would originate from the two LSPs in the final states of the gluino and squark decays, assuming $R$-parity conservation.

This analysis required at least three jets satisfying $E_{T}^{(1)}>70 \mathrm{GeV}, E_{T}^{(2)}>$ $30 \mathrm{GeV}$, and $E_{T}^{(3)}>15 \mathrm{GeV}$, and also required $\not_{T}>70 \mathrm{GeV}$. Also, the three highest $E_{T}$ jets were required not to be near the $\mathbb{F}_{T}$ direction. In addition, $H_{T} \equiv E_{T}^{(2)}+E_{T}^{(3)}+\not_{T}>150 \mathrm{GeV}$ was required for the expected signal region. Events with large $\not_{T}$ and $\geq 3$ jets in the final state were expected primarily from QCD, $Z(\rightarrow \nu \nu)+\geq 3$ jets, $W(\rightarrow \tau \nu)+2$ jets, and $t \bar{t}$ processes. We expected $76 \pm 13$ events from the standard model processes and observed 74 events. The $\not_{T}$ and $H_{T}$ requirements were then optimised to increase the 
sensitivity to the signal in the context of MSSM and mSUGRA models in the $m_{\tilde{q}}-m_{\tilde{g}}$ plane. The resulting $95 \%$ CL limit is shown in Fig. 2. This search excludes $m_{\tilde{g}}<190 \mathrm{GeV} / c^{2}$, independent of $m_{\tilde{q}}$, and $m_{\tilde{g}}<300 \mathrm{GeV} / c^{2}$ in the case of $m_{\tilde{g}} \simeq m_{\tilde{q}}$.

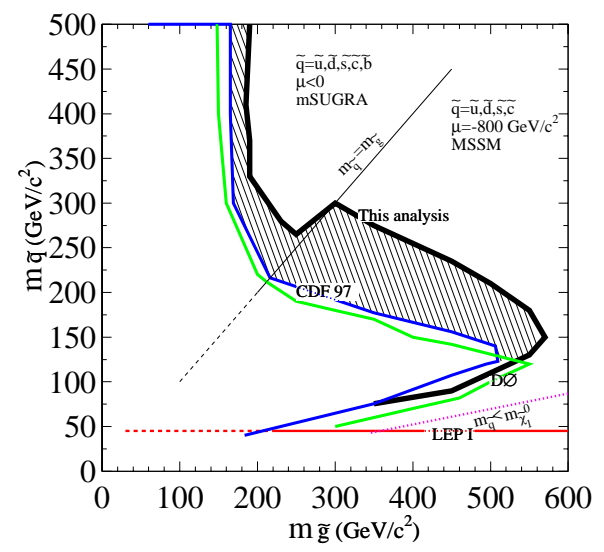

Fig. 2. The $95 \%$ confidence level limit curve in the $m_{\tilde{q}}-m_{\tilde{g}}$ plane got $\tan \beta=3$. The hatched area is newly excluded by this analysis. Results from previous searches are also shown

\section{Scalar Top Quark Searches}

\subsection{R-Parity Conserving Stop Decay}

This analysis searched for a pair production of scalar top quarks (stops) decaying through a $R$-parity conserving process [21]. For the very heavy charged sleptons, it was assumed that the stop decays only to $b l \tilde{\nu}(l=e, \mu, \tau)$. The signature searched for was two oppositely charged leptons, $\not_{T}>15 \mathrm{GeV}$, and $\geq 1$ jets, where $\not_{T}$ comes from $\tilde{\nu} \overline{\tilde{\nu}}$.

After the further selection requirements optimised for small mass difference between the stop and sneutrino, the expected number of backgrounds was $1.52 \pm 0.26$ (stat.) \pm 0.32 (syst.) events mostly coming from lepton misidentification, $Z(\rightarrow l l)+$ jets, and $t \bar{t}$ production. No events survived the selection cuts and the $95 \%$ CL exclusion region in the $m_{\tilde{\nu}}-m_{\tilde{t}}$ plane was calculated as shown in Fig. 3.

\subsection{R-Parity Violating Stop Decay}

This analysis searched for a stop pair production in which a stop decays into $\tau b$ through $R$-parity violating processes [22]. Assuming that either the stop 


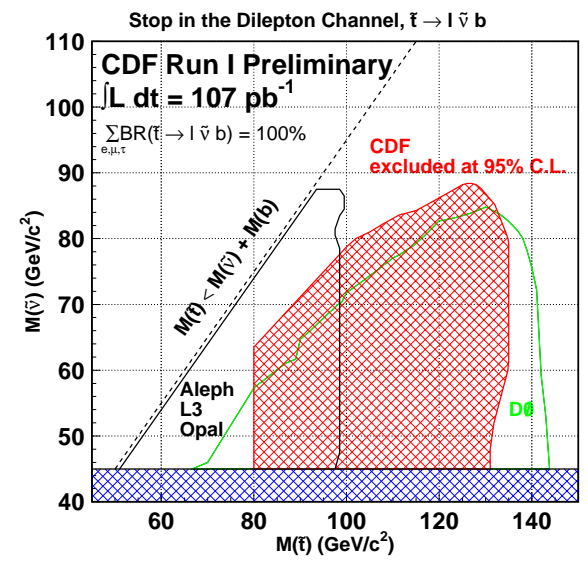

Fig. 3. The $95 \% \mathrm{CL}$ exclusion region in $m_{\tilde{\nu}}-m_{\tilde{t}}$ plane for $\tilde{t} \rightarrow l \tilde{\nu} b$ for the $R$-parity conserving supersymmetry

is the LSP or the dominant decay mode for stop is $\tilde{t} \rightarrow \tau b$ when the stop is NLSP, the stop is expected always to decay to $\tau b$.

The final state searched for was $l \nu_{l} \nu_{\tau} \tau_{h} b b(l=e, \mu)$ where one $\tau$ decays leptonically and the other decays hadronically $\left(\tau_{h}\right)$. An electron (muon) was required to have $E_{T}>10 \mathrm{GeV}\left(p_{T}>10 \mathrm{GeV} / c\right)$ and $\tau_{h}$ was identified by a track based $\tau_{h}$ identification including $\pi_{0}$ reconstruction. In order to reduce the background further, it was required that the transverse mass of $\left(l, \not_{T}\right)$ be $<35 \mathrm{GeV}, E_{T}^{e}\left(p_{T}^{\mu}\right)+\not_{T}+p_{T}\left(\tau_{h}\right)>75 \mathrm{GeV}$, and two or more jets.

The expected number of background was $3.2_{-0.3}^{+1.4}$ dominated by $Z(\rightarrow$ $\tau \tau)+$ jets and no candidate event was found. This analysis excluded at $95 \%$ CL the $\tilde{t} \bar{t}$ production cross section as a function of stop mass. The results are shown in Fig. 4. The 95\% CL lower mass limits for stop was set at $119 \mathrm{GeV} / c^{2}$.

\section{References}

1. T. Affolder et al., CDF Collaboration, Phys. Rev. Lett. 87, 231803 (2001).

2. F. Abe et al., CDF Collaboration, Phys. Rev. Lett. 84, 5716 (2000);

3. F. Abe et al., CDF Collaboration, hep-ex/020930 (2002).

4. F. Abe et al., CDF Collaboration, Phys. Rev. Lett. 79, 2191 (1997).

5. CDF Collaboration, Internal Note 6080 (2002).

6. R. Blair et al., CDF Collaboration, FERMILAB-PUB-96/390-E (1996).

7. T. Affolder et al., CDF Collaboration, Phys. Rev. Lett. 85, 2062 (2000).

8. T. Han, J. Lykken, and R. Zhang, Phys. Rev. D 59, 105006 (1999).

9. G. F. Guidice, R. Rattazzi, and J. D. Wells, Nucl. Phys. B544, 3 (1999).

10. CDF Collaboration, Internal Note 5694 (2001); J. M. Carlson, Ph.D. thesis, University of Michigan (2002). 


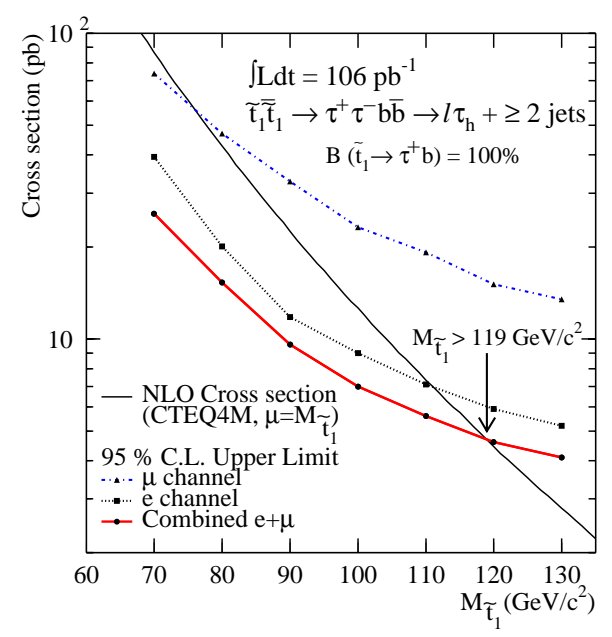

Fig. 4. The 95\% CL limits on the production cross section of the stop through $R$-parity violating decay, $\tilde{t}_{1} \overline{\tilde{t}}_{1} \rightarrow \tau^{+} b \tau^{-} \bar{b}$, and the theory calculation as a function of its mass. The regions above the curves are excluded

11. J. L. Hewett, Phys. Rev. Lett. 82, 4765 (1999).

12. L. Randall and R. Sundrum, Phys. Rev. Lett. 83, 3370 (1999).

13. D. Acosta et al., CDF Collaboration, FERMILAB-PUB-02/119-E (2002).

14. A. Connolly for the CDF Collaboration, FERMILAB-CONF-99/092-E (1999).

15. F. Abe et al., CDF Collaboration, Phys. Rev. Lett. 79, 4327 (1997).

16. F. Abe et al., CDF Collaboration, Phys. Rev. Lett. 81, 4806 (1998); CDF Collaboration, Internal Note 5790 (2001).

17. F. Abe et al., CDF Collaboration, Phys. Rev. Lett. 78, 2906 (1997); T. Affolder et al., CDF Collaboration, Phys. Rev. Lett. 85, 2056 (2000).

18. F. Abe et al., CDF Collaboration, Phys. Rev. D 59, 092002 (1999).

19. D. Acosta et al., CDF Collaboration, Phys. Rev. Lett. 89, 041802 (2002).

20. T. Affolder et al., CDF Collaboration, Phys. Rev. Lett. 88, 041801 (2002).

21. CDF Collaboration, Internal Note 6171 (2002); A. Pompos, Ph.D. thesis, Purdue University (2002).

22. J. Zhou for the CDF Collaboration, hep-ex/0205098 (2002). 